\title{
Ultrafast interferometric measurements of plasmonic transport in photonic crystals
}

\author{
Yish-Hann Liau \\ Department of Chemistry, The James Franck Institute, University of Chicago, 5735 South Ellis Avenue, Chicago, Illinois 60637
} Shunji Egusa

Department of Physics, University of Chicago, 5735 South Ellis Avenue, Chicago, Illinois 60637

Norbert F. Scherer

Department of Chemistry, The James Franck Institute, University of Chicago, 5735 South Ellis Avenue, Chicago, Illinois 60637

Received January 3, 2002

\begin{abstract}
We present a novel time-domain experimental approach to the study of the dynamics of surface electromagnetic wave propagation in a two-dimensional photonic crystal. A surface plasmon polariton is launched by ultrafast laser pulses and propagates into a photonic crystal, the dynamics of which are measured by an interferometric cross-correlation method. Plasmon photonic stopgaps are characterized by a single measurement. The dispersion around the stopgaps is determined with a series of angle-resolved measurements. (C) 2002 Optical Society of America

OCIS codes: $240.6680,320.7150,260.6970$.
\end{abstract}

Photonic crystal structures, which typically consist of materials with periodically varying dielectric properties, ${ }^{1-3}$ show great promise for use in the manipulation of light to an unprecedented degree. If the Bragg condition is fulfilled, photonic bandgaps can be created. A photonic bandgap in all propagation directions would prohibit even spontaneous emission of an atom inside a photonic crystal. ${ }^{2}$

The goal of manipulating light on subwavelength scales requires a different approach to break the diffraction limit that sets the fundamental length scale of optical devices. A surface plasmon polariton (SPP) is collective electron excitation that is spatially confined at the metal-air interface ${ }^{4}$ and propagates as a longitudinal wave. Resonant transduction of an optical field into the SPP mode of the medium and the reverse can occur as long as energy and momentum are simultaneously conserved. ${ }^{4}$ Therefore, transducing light into the SPP mode may be the most feasible approach to optical manipulation on subwavelength and even nanometer scales.

Understanding the dynamics of the interaction of light with a photonic structure requires both amplitude and phase characterization. Conversely, complete information about the propagating field permits characterizing the photonic crystal's dielectric properties and structures. There have been numerous studies of photonic crystals made with cw light ${ }^{5-7}$; nevertheless, time-resolved studies of photonic crystals are almost nonexistent. ${ }^{8,9}$ Although time- and frequency-domain representations of light are equivalent, provided that both the amplitude and the phase are fully characterized, the majority of frequencydomain experiments are intensity-based measurements, thus having lost the phase information. Further, the use of high peak-intensity femtosecond pulses creates possibilities to exploit the optical nonlinearity of photonic materials ${ }^{10}$ leading to new approaches to all-optical manipulation of light.
In this Letter we present a time-domain approach to the study of the transport of a confined surface wave in a two-dimensional photonic crystal. The experiments build on earlier studies of surface-wave propagation at periodically decorated metallic surfaces ${ }^{11}$ and SPP coherent multiple scattering. ${ }^{12}$ The dynamics of a SPP propagating in a photonic structure is directly observed by interferometric correlation measurements. The broad bandwidth of the ultrashort pulses makes it possible to identify plasmon stopgaps in a single measurement and to construct a dispersion curve about these stopgaps from a series of angle-resolved measurements.

The laser system and the experimental setup have been described elsewhere, ${ }^{12,13}$ so only their key aspects are summarized. The femtosecond laser pulse train from a home-built cavity-dumped Ti:sapphire oscillator was sent to a noncollinear Mach-Zehnder interferometer to generate two pump-probe pulse pairs with a controlled time delay. One pulse pair, focused into and recombined by a moiré grating, was used to generate a reference interferogram. The other pair of $p$-polarized beams was directed and focused to the hypotenuse of a hemispheric prism at the appropriate incident angle to launch the SPP. The attenuated total internal reflection and the conical emission that occur at the resonant angle for smooth metal films indicate SPP excitation. ${ }^{4}$ A plasmonic photonic crystal was fabricated by decoration of a thin Ag film with a monolayer of polystyrene beads. Because the refractive index of polystyrene is significantly larger than that of air ( $n=1.59$ and $n=1$, respectively), the surface plasmon was strongly scattered as it encountered the Ag film-polystyrene triangular lattice.

The sample shown in Fig. 1A consisted of a monolayer of closely packed spheres (right-hand two thirds of the image) on a smooth Ag film. The image in Fig. 1B was obtained when the laser beam was focused in the smooth film region. The propagating SPP was 

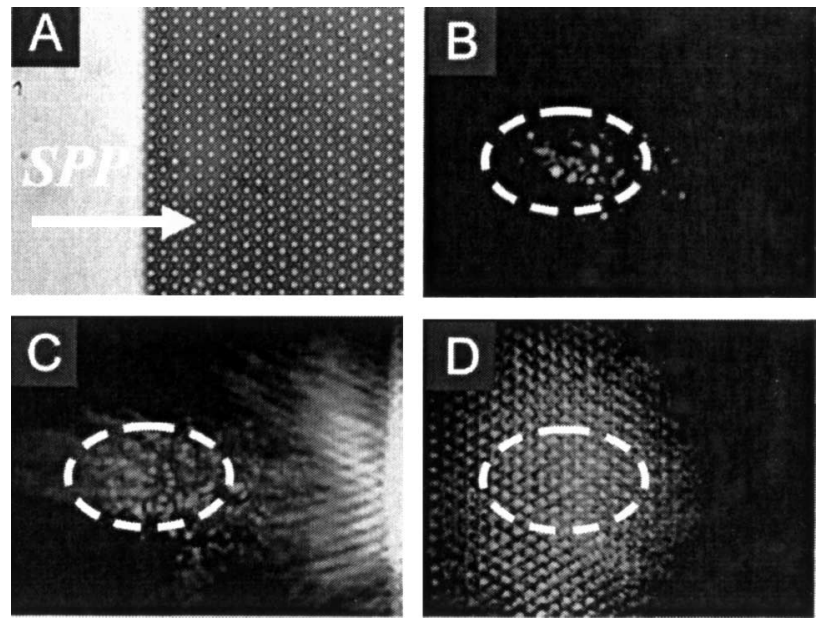

Fig. 1. A, Optical microscopy image of the plasmon photonic crystal. The sample, consisting of a monolayer of 2.1- $\mu \mathrm{m}$-diameter latex spheres, covers only the right-hand portion of the field of view and forms a straight boundary. Image area, $44 \mu \mathrm{m}$ by $60 \mu \mathrm{m}$. B-D, Bragg scattering of surface plasmons by the lattice structure. The plasmon is launched at the position indicated by the dashed ovals and propagates into the crystal indicated by the arrow A. $\mathrm{B}$, laser beam focused to the smooth film area; C, the edge of the lattice is away from the launching position of the plasmon but still within the propagation length; $\mathrm{D}$, the laser beam is focused onto the perfect-lattice region.

scattered by surface roughness, yielding the bright spots. Figure 1C shows the optical scattering from the lattice boundary located at the right-hand edge of the field of view; a standing-wave pattern is clearly observable. The distance from the boundary to the center of focus is significantly larger than the focal spot, clearly showing that the SPP propagates to and scatters from the lattice. The reflected plasmon field interferes with the incident plasmon and forms the standing-wave pattern shown in the image. The standing-wave pattern possesses clear directionality owing to the triangular symmetry of the lattice. As the lattice is moved closer to the launching point of the SPP the scattering becomes stronger and the plasmon penetrates further into the lattice (data not shown). The image in Fig. 1D is obtained by direction of the laser to the perfect lattice region. Interestingly, the propagation was shorter along the forward direction than orthogonally to it and much shorter than the propagation distance in Fig. 1C or in simple Ag film. The lattice inhibits the forward propagation of the plasmon field but at this wavelength permits relatively more lateral propagation; the inhibited propagation results from the plasmon stop band created by the lattice.

The pump-probe interferograms of Fig. 2 are the cross correlation between the pump and the plasmon fields (i.e., the impulse response function of the photonic crystal) and an (essentially unmodified) "idler" beam. Figure 2A shows the interferogram obtained when the pump-probe beams are directed to the perfect-lattice region. The signal shows complex constructive and destructive interference patterns, indicating rapid variation of the temporal phase that results from multiple scattering and the superposition of different propagation distances of the SPP in the crystal. The measured interferometric response shows recurrent patterns that lasted as long as several hundred femtoseconds; the decay of the patterns indicates a loss of amplitude (and coherence) as a result of propagation in directions outside the detection angle or from radiative scattering from the film. The small imaginary part of the Ag dielectric constant also contributes slightly to the decay. The temporal pattern of the measured signal depends strongly on the magnitude and the direction of the SPP wave vector with respect to the lattice direction, as we observed by monitoring the angle-resolved interferogram and by rotating the lattice. Figure $2 \mathrm{~B}$ shows the interferogram measured when the pump-probe pair was focused in the smooth film region. Figure $2 \mathrm{C}$ shows the reference interferogram measured simultaneously with that in Fig. 2A (the same zero of time and phase relationship).

Qualitatively different modulated interferograms were obtained from analogous pump-probe measurements from a monolayer of latex spheres upon glass (i.e., without the $\mathrm{Ag}$ film). The signals reported here result from SPP propagation as verified by a wave-vector dependence on the attenuated total reflection resonance and by the observation of modulated interferograms for the $\mathrm{Ag}$ film photonic crystals even when the pump-probe focus was in the front of the crystal, as shown in Fig. $2 \mathrm{C}$, a situation that necessitating plasmon propagation over several tens of micrometers.

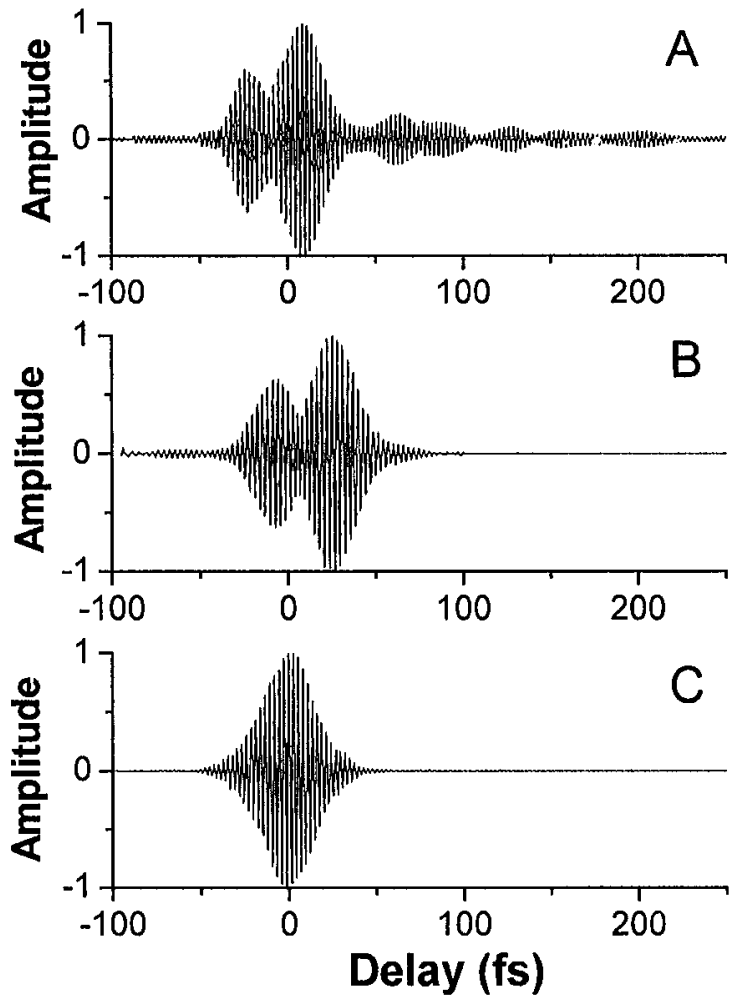

Fig. 2. A, Representative pump-modulated lock-in amplifier demodulated interferometric signals measured from $A$, the perfect-lattice region and $B$, the metal film. $\mathrm{C}$, Simultaneously measured reference interferogram. 


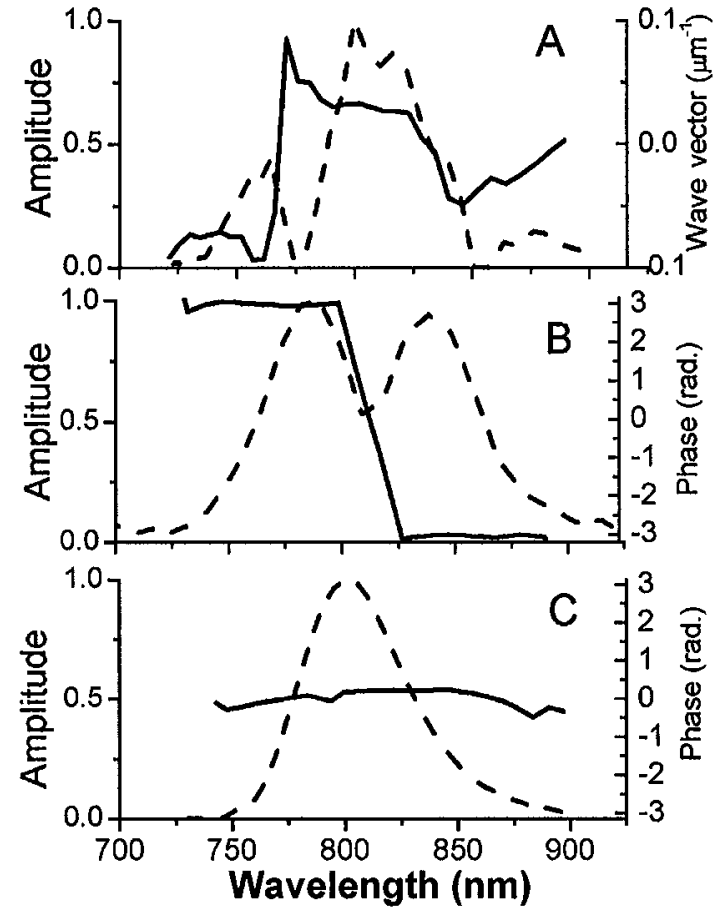

Fig. 3. Spectral amplitude and spectral phase of the interferogram obtained from A, the plasmonic photonic crystal, B, the metal film, and $\mathrm{C}$, the reference. Solid curves, truncated spectral phase or wave vector of ultrafast laser pulses measured from the sample or reference. Dashed curves, spectral amplitudes.

The data were analyzed through the complex response function obtained by Fourier transformation, defined $\operatorname{as}^{8} H(\omega)=A(\omega) \exp [i \varphi(\omega)]$, where $A(\omega)$ and $\varphi(\omega)$ are the spectral amplitude and the spectral phase, respectively. The phase is related to propagation length $L$ and to wave vector $k(\omega)$ by $\varphi(\omega)=k(\omega) L$, and the amplitude reflects the spectral (hence the energy) content of the optical field. The group velocity of the SPP, $\nu_{g}\left(\omega_{0}\right)=[\mathrm{d} k(\omega) / \mathrm{d} \omega]_{\omega_{0}}^{-1}$, on $\mathrm{Ag}$ films is $\sim 0.28 \mu \mathrm{m} / \mathrm{fs}$ (i.e., $0.93 \mathrm{c}$ ) determined from the dielectric constant of $\mathrm{Ag}$ at a carrier (angular) frequency of $2.356 \times 10^{15} \mathrm{~Hz}^{14}$ Assuming that the SPP propagates with the same velocity in the crystal region as it does in the smooth $\mathrm{Ag}$ film allows the propagation length in the photonic crystal to be calculated and used to scale the spectral phase function to yield the frequency-dependent wave vector.

The results of this analysis are shown in Fig. 3. There are clearly two dips in the spectral amplitude (Fig. 3A) that result from the splitting of the dispersion curve. Two spectral features within the bandwidth of the laser are resonant with the modulated Ag film dispersion relation and propagate in the photonic crystal film. The intervening peak corresponds to a stop band for the plasmon; frequency components in this region are not allowed to propagate. This observation is in contrast to the case of smooth Ag films in which only one dip is observed (Fig. 3B), corresponding to a single-valued dispersion relation.

The plasmonic field is strongly modified by the photonic crystal, which results in abrupt changes in its wave vector. Figure 3A (solid curve) shows the largest variations at the same frequencies as the dips in the spectrum. This result is quite different from that for the single-valued dispersion-resonance observed for the $\mathrm{Ag}$ thin film (Fig. 3B). The wave vector of the field associated with the reference is essentially a flat horizontal line (Fig. 3C, solid curve), and the spectral amplitude recovers the laser spectrum (Fig. 3C, dashed line). We will show elsewhere that the dispersion of the two-dimensional plasmonic photonic crystals can be obtained by a series of angle-resolved interferograms.

In conclusion, a new approach to the study of the dynamics of a surface plasmon polariton in photonic crystals by ultrafast interferometric measurements has been presented. The high spatial confinement of the SPP mode relative to the incident optical field is a promising route to optical propagation in nanophotonic structures. ${ }^{15,16}$ The complete field characterization and analysis approach described here will be an important step in refining the design and function of such materials.

This research was supported by the W. M. Keck Foundation and the University of Chicago Materials Research Science and Engineering Center. We thank Michael Martin for assistance with some of the measurements. N. F. Scherer's e-mail address is nfschere@uchicago.edu.

\section{References}

1. S. John, Phys. Rev. Lett. 53, 2169 (1984).

2. E. Yablonovitch, Phys. Rev. Lett. 58, 2059 (1987).

3. J. D. Joannopoulos, R. D. Meade, and J. N. Winn, Photonic Crystals (Princeton U. Press, Princeton, N.J., 1995).

4. H. Raether, Surface Plasmons on Smooth and Rough Surfaces and on Gratings (Springer-Verlag, Berlin, 1988).

5. I. I. Tarhan and G. H. Watson, Phys. Rev. Lett. 76, 315 (1996).

6. S. C. Kitson, W. L. Barnes, and J. R. Sambles, Phys. Rev. Lett. 77, 2670 (1996).

7. T. W. Ebbesen, H. J. Lezec, H. F. Ghaemi, T. Thio, and P. A. Wolff, Nature 391, 667 (1998).

8. A. Imhof, W. L. Vos, R. Sprik, and A. Lagendijk, Phys. Rev. Lett. 83, 2942 (1999).

9. S. Wang, H. Erlig, H. R. Fetterman, E. Yablonovitch, V. Grubsky, D. S. Starodubov, and J. Feinberg, Microwave Opt. Technol. Lett. 20, 17 (1999).

10. V. Berger, Phys. Rev. Lett. 81, 4136 (1998).

11. R. H. Ritchie, E. T. Arakawa, J. J. Cowan, and R. N. Hamm, Phys. Rev. Lett. 21, 1530 (1968).

12. W. Wang, M. Feldstein, and N. F. Scherer, Chem. Phys. Lett. 262, 573 (1996).

13. Y.-H. Liau, A. N. Unterreiner, D. C. Arnett, and N. F. Scherer, Appl. Opt. 38, 7386 (1999).

14. E. D. Palik, Handbook of Optical Constants of Solids (Academic, San Diego, Calif., 1985).

15. R. M. Dickson and L. A. Lyon, J. Phys. Chem. B 104, 6095 (2000).

16. S. A. Maier, M. L. Brongersma, P. G. Kik, S. Meltzer, A. G. Requicha, and H. A. Atwater, Adv. Mater. 13, 1501 (2001). 